\title{
BIRKHOFF NORMAL FORMS FOR BORN-OPPENHEIMER OPERATORS
}

\author{
NAWEL LATIGUI ${ }^{1,4}$, BEKKAI MESSIRDI ${ }^{2,4, *}$ AND KAOUTAR GHOMARI $^{3,4}$ \\ ${ }^{1}$ Department of Mathematics, Faculy of Exact and Applicable Sciences, University of Oran1 Ahmed Ben \\ Bella, Algeria \\ ${ }^{2}$ High School of Electrical Engineering and Energetics-Oran, Algeria \\ ${ }^{3}$ Department of Mathematics and Informatics, ENPOran, Algeria \\ ${ }^{4}$ Laboratory of Fundamental and Applicable Mathematics of Oran (LMFAO), Algeria \\ ${ }^{*}$ Corresponding author: bmessirdi@yahoo.fr
}

\begin{abstract}
We describe in this paper a significant spectral reduction method for Born-Oppenheimer operators with regular potentials, which leads to an adaptable Birkhoff normal form theorem for the associated effective Hamiltonians. As illustration of the established results, we compute the Birkhoff normal form in Fermi resonance.
\end{abstract}

\section{INTRODUCTION}

For a molecular system with $N$ electrons and $N^{\prime}$ nuclei, the Hamiltonian, under the Born-Oppenheimer approximation, can be written as:

$$
P(h)=-h^{2} \Delta_{x}+Q(x), Q(x)=-\Delta_{y}+V(x, y)
$$

on $L^{2}\left(\mathbb{R}_{x}^{n} \times \mathbb{R}_{y}^{p}\right)$ where $n=3 N$ and $p=3 N^{\prime}$ and where $h>0$ is a small parameter playing the role of the semi-classical parameter. $\Delta_{x}$ (resp. $\Delta_{y}$ ) is the Laplace operator with respect to $x$ (resp. $y$ ), $x \in \mathbb{R}^{n}$ and $y \in \mathbb{R}^{p}, N, N^{\prime} \geq 1, V$ is the interaction potential between particles. $P(h)$ is called the Born-Oppenheimer

Received 2019-10-12; accepted 2019-11-11; published 2020-03-02.

2010 Mathematics Subject Classification. 58K50, 81S10, 47A20.

Key words and phrases. Born-Oppenheimer operator; effective Hamiltonian; Birkhoff normal form; harmonic oscillator; Fermi resonance.

(C)2020 Authors retain the copyrights of their papers, and all open access articles are distributed under the terms of the Creative Commons Attribution License. 
Hamiltonian. $Q(x)$ is the electronic Hamiltonian defined on $L^{2}\left(\mathbb{R}_{y}^{p}\right)$. It is well known that if $V(x, y)$ is smooth verifying suitable assumptions, then

$$
z \in \sigma(P(h)) \Longleftrightarrow z \in \sigma(F(z))
$$

where $F(z)$ is a semiclassical analytic pseudodifferential operator on $L^{2}\left(\mathbb{R}_{x}^{n}\right)$ and $\sigma$ stands for the spectrum, (see e.g. [10], [11], [3]), the main idea, due to Born and Oppenheimer in [5], is to replace, for fixed $x$, the operator $Q(x)$ by its eigenvalues. This reduction is possible thanks to the pseudodifferential calculus with operator valued symbols. Then we are led to consider, the reduced operator (called the effective Hamiltonian in the Born-Oppenheimer approximation):

$$
P_{e f f}(h)=-h^{2} \Delta_{x}+\lambda_{1}(x)
$$

where $\lambda_{1}(x)$ is the lowest eigenvalue of $Q(x)$, by the minimax principle $\lambda_{1}(x)$ is simple and analytic if $V$ is sufficiently smooth. Motivated by various physical questions we consider the connected problems in the asymptotic $h \rightarrow 0^{+}$, note that through standard semiclassical analysis $P_{\text {eff }}(h)$ can explain the complete spectral picture of $P(h)$ modulo errors in $h$.

We wish to describe the Birkhoff normal form near an equilibrium point of $P(h)$. It is well known that a more precise description of the vibrational energies of a molecule is given by the harmonic oscillator, our approach here is to replace $Q(x)$ in the Born-Oppenheimer Hamitonian $P(h)$ by its lowest eigenvalue $\lambda_{1}(x)$ and thus, we are reduced to an effective $h$-pseudodifferential operator $O p\left(e_{\lambda}\right)$, with symbol $e_{\lambda}$ depending only on $(x, \xi)$. The normal forms in the Born-Oppenheimer approximation, are introduced here as being those of the Schrödinger effective operator $P_{\text {eff }}(h)$ on $L^{2}\left(\mathbb{R}_{x}^{n}\right)$. Birkhoff normal form is one of the basic tools in quantum and semiclassical mechanics (see e.g. [7], [8]), it has already been used by Birkhoff [4] to study some problems of dynamical systems.

Precisely, the goal of this paper is to analyze the notion of the Birkhoff normal form near an equilibrium point and discuss the dynamical consequences for the Schrödinger Hamiltonian $P(h)$. Suppose 0 is a nondegenerate local minimum of $\lambda_{1}(x)$, by applying semiclassical techniques especially the pseudodifferential calculus with operator valued symbols and the classical quantum formal Birkhoff normal form theorem, we show that we can find a canonical transformation putting $P(h)$ as a reasonable perturbation of $-\Delta_{x}+$ $\frac{1}{2}\left\langle\lambda_{1}^{\prime \prime}(0) x, x\right\rangle$ modulo $\mathcal{O}\left(h^{2}\right)$. Our approach is natural, it consists in computing the normal form of the effective Hamiltonian $-h^{2} \Delta_{x}+\lambda_{1}(x)$ after reduction of the operator $P(h)$ to $P_{e f f}(h)$. To our knowledge this is the first attempt to determine the Birkoff normal forms for the Born-Oppenheimer Hamiltonians.

In Section 2, we recall some results on pseudodifferential operators with operator valued symbols. Then, we give a representation of the effective Hamiltonian and obtain WKB solutions of the Hamiltonian $P(h)$. In section 3, we investigate the theorem of Birkhoff normal form near an equilibrium point in infinite 
dimension in the Born-Oppenheimer approximation for $P(h)$ via the effective Hamiltonian $P_{\text {eff }}(h)$. In the fourth section we compute the Birkhoff normal form of $P_{\text {eff }}(h)$ in the Fermi resonance.

\section{REDUCTION TO AN EFFECTIVE OpERATOR}

In this section we explain the construction of WKB solutions for the Hamiltonian $P(h)$ and several mathematical results concerning the pseudodifferential calculus with operator valued symbols of the BornOppenheimer approximation. For further informations about the pseudodifferential calculus and BKW method we refer the reader to the works of Balazard-Konlein [2], Messirdi [10], Baklouti [1] and other authors.

2.1. Pseudodifferential calculus with operator valued symbols. For $m \in \mathbb{R}, \Omega$ a bounded open subset of $\mathbb{R}_{x}^{n}$ and $H$ a complex Hilbert space, consider the space of formal power series:

$$
S^{m}(\Omega, H)=\left\{\sum_{j=0}^{\infty} h^{-m+j / 2} s_{j}(x): s_{j} \in C^{\infty}(\Omega, H)\right\}
$$

where $C^{\infty}(\Omega, H)$ is the space of $C^{\infty}$-functions mapping $\Omega$ into $H$.

Given $\psi \in C^{\infty}(\Omega, \mathbb{R})$ and $\mathcal{U}$ a neighborhood of 0 in $\mathbb{R}_{x}^{n}$, we set:

$$
\Omega^{*}=\left\{(x, \xi) \in \Omega \times \mathbb{C}^{n}: \xi-i \nabla \psi(x) \in \mathcal{U}\right\}
$$

and

$$
S^{0}\left(\Omega^{*}, \mathcal{L}(H, K)\right)=\left\{\sum_{j=0}^{\infty} h^{j} a_{j}(x, \xi): a_{j} \in C^{\infty}\left(\Omega^{*}, \mathcal{L}(H, K)\right)\right\}
$$

where $K$ is Hilbert space and $\mathcal{L}(H, K)$ is the algebra of all continuous linear operators from $H$ into $K$. The operator valued functions in $S^{0}\left(\Omega^{*}, \mathcal{L}(H, K)\right)$ are called symbols.

For any symbol $a=a(x, \xi ; h)$ in $S^{0}\left(\Omega^{*}, \mathcal{L}(H, K)\right)$, by analogy with the action of differential operators on the space $e^{-\psi(x) / h} S^{m}(\Omega, H)$, one can define an operator $O p(a)$ from $e^{-\psi(x) / h} S^{m}(\Omega, H)$ into $e^{-\psi(x) / h} S^{m}(\Omega, K)$ by the formula:

$$
\begin{gathered}
O p(a)\left(e^{-\psi(x) / h} s(x, h)\right)= \\
e^{-\psi(x) / h} \sum_{\alpha \in \mathbb{N}^{n}} \frac{h^{|\alpha|}}{i^{|\alpha|} \alpha !} \partial_{\xi}^{\alpha} a(x, i \nabla \psi(x) ; h) \partial_{y}^{\alpha}\left(s(y, h) e^{\chi(x, y) / h}\right)_{y=x} \\
\chi(x, y)=\psi(y)-\psi(x)-(y-x) . \nabla \psi(x)=\mathcal{O}\left(|x-y|^{2}\right), s \in S^{m}(\Omega, H) .
\end{gathered}
$$

$O p(a)$ is called $h$-pseudodifferential operator with operator valued symbol $a(x, \xi ; h)=\sum_{j=0}^{\infty} h^{j} a_{j}(x, \xi)$. The function $a_{0}(x, \xi)$ (coefficient of $h^{0}$ ) is called principal symbol of the $h$-pseudodifferential operator $O p(a)$. Furthermore, such operators verify:

$$
e^{\psi(x) / h} O p(a)\left(e^{-\psi(x) / h} s(x, h)\right) \in S^{m}(\Omega, H)
$$


and can be composed using the formula:

$$
O p(b) \circ O p(a)=O p(b \sharp a)
$$

where $a \in S^{0}\left(\Omega^{*}, \mathcal{L}(H, K)\right), b \in S^{0}\left(\Omega^{*}, \mathcal{L}(K, L)\right)$ ( $L$ is a third Hilbert space), the range of $O p(a)$ is contained in the domain of $O p(b)$, and

$$
b \sharp a(x, \xi, h)=\sum_{\alpha \in \mathbb{N}^{n}} \frac{h^{|\alpha|}}{i^{|\alpha|} \alpha !} \partial_{\xi}^{\alpha} b(x, \xi ; h) \partial_{x}^{\alpha} a(x, \xi ; h) \in S^{0}\left(\Omega^{*}, \mathcal{L}(H, L)\right) .
$$

This formula makes it possible to inverse asymptotically operators $O p(a)$ whose principal symbol $a_{0}(x, \xi)$ is invertible as a linear operator from $H$ into $K$.

2.2. BKW solutions (scalar case). Let us take $H=\mathbb{C}$ and recall the following result:

Theorem 2.1. ([9]) Let $a(x, \xi ; h)=\sum_{j=0}^{\infty} h^{j} a_{j}(x, \xi) \in S^{0}\left(\Omega^{*}, \mathbb{C}\right)$ be such that $a_{0}(x, \xi)=\xi^{2}+\lambda(x)$ where $\lambda \in C^{\infty}(\Omega, \mathbb{R}), \lambda \geq 0, \lambda^{-1}(0)=\{0\}, \lambda^{\prime}(0)=0$ and $\lambda^{\prime \prime}(0)>0$. Let $C_{0}>0$ and $N_{0}$ be the number of eigenvalues of

$$
-\Delta_{x}+\frac{1}{2}\left\langle\lambda^{\prime \prime}(0) x, x\right\rangle
$$

in the compact interval $\left[0, C_{0}\right]$. Denote by $e_{1}, \ldots, e_{N_{0}}$ these eigenvalues. Then there are formal series:

$$
E_{k}(h)=e_{k} h+\sum_{j=1}^{\infty} e_{k, j} h^{1+j / 2} \text { and } a_{k}(x, h) \in S^{m_{k}}(\Omega, \mathbb{C})
$$

$e_{k, j}, m_{k} \in \mathbb{R}, k \in\left\{1, \ldots, N_{0}\right\}$, such that

$$
\left(O p(a)-E_{k}(h)\right)\left(e^{-\psi(x) / h} a_{k}\right)=0 \text { in } e^{-\psi(x) / h} S^{m_{k}}(\Omega, \mathbb{C})
$$

where $\psi(x)$ is the Agmon distance associated to the metric $\lambda(x) d x^{2}$. The functions $e^{-\psi(x) / h} a_{k}(x, h)$ are called the BKW solutions.

2.3. BKW solutions (general case). Let $V \in C^{\infty}\left(\Omega, \mathcal{L}\left(H^{2}\left(\mathbb{R}_{y}^{p}\right), L^{2}\left(\mathbb{R}_{y}^{p}\right)\right)\right)$ be $\Delta$-compact:

$$
V(x, y)\left(-\Delta_{y}+1\right)^{-1} \in C^{\infty}\left(\Omega, \mathcal{L}\left(L^{2}\left(\mathbb{R}_{y}^{p}\right)\right)\right)
$$

where $\Omega$ is a bounded open subset of $\mathbb{R}_{x}^{n}$. Thus, $P(h)$ is selfadjoint on $L^{2}\left(\mathbb{R}_{x}^{n} \times \mathbb{R}_{y}^{p}\right)$ with domain $H^{2}\left(\mathbb{R}_{x}^{n} \times \mathbb{R}_{y}^{p}\right)$ as well as the operator $Q(x)$ on $L^{2}\left(\mathbb{R}_{y}^{p}\right)$ with domain $H^{2}\left(\mathbb{R}_{y}^{p}\right)$. Denote

$$
\lambda_{1}(x)=\inf (\sigma(Q(x)))
$$

the lowest energy level (ground state) of operator $Q(x)$. Suppose that $\lambda_{1}(x)$ is an isolated eigenvalue of finite multiplicity of $Q(x)$, having unique and non-degenerate minimum at 0 :

$$
\lambda_{1}(x) \geq 0, \lambda_{1}^{-1}(0)=\{0\}, \lambda_{1}^{\prime}(0)=0, \lambda_{1}^{\prime \prime}(0)>0,
$$


and that $\lambda_{1}(x)$ is separated from the rest of the spectrum $\sigma(Q(x))$, i.e.,

$$
\inf _{x \in \mathbb{R}^{n}}\left(\inf \left(\sigma(Q(x)) \backslash\left\{\lambda_{1}(x)\right\}\right)\right)>0
$$

We also denote by $u_{1}(x, y)$ the first eigenfunction of $Q(x)$ associated with $\lambda_{1}(x)$ and normalized by $\left\|u_{1}(x, .)\right\|_{L^{2}\left(\mathbb{R}_{y}^{p}\right)}=1$ for all $x \in \mathbb{R}^{n}$. It can be shown that $\lambda_{1} \in C^{\infty}(\Omega, \mathbb{R})$ and $u_{1} \in C^{\infty}\left(\Omega, H^{2}\left(\mathbb{R}_{y}^{p}\right)\right)(\mathrm{cf}$. [10]). In particular, the assumption (2.4) implies that the orthogonal projection $\Pi(x)$ on the subspace of $L^{2}\left(\mathbb{R}_{y}^{p}\right)$ spanned by $u_{1}(x,),. x \in \Omega$, is $C^{2}$-regular with respect to $x$ (see [6]). To construct BKW solutions of $P(h)$, the idea here is to use the pseudodifferential calculus with operator valued symbols developed in subsection 2.1 .

Consider, for $\lambda \in \mathbb{C}$, the following symbol:

$$
a_{\lambda}(x ; \xi)=\left(\begin{array}{cc}
\xi^{2}+Q(x)-\lambda & u_{1} \\
\left\langle., u_{1}\right\rangle_{y} & 0
\end{array}\right) \in S^{0}\left(\Omega^{*}, \mathcal{L}\left(H^{2}\left(\mathbb{R}_{y}^{p}\right) \oplus \mathbb{C}, L^{2}\left(\mathbb{R}_{y}^{p}\right) \oplus \mathbb{C}\right)\right)
$$

where $\left\langle., u_{1}\right\rangle_{y}$ is the inner product in $L^{2}\left(\mathbb{R}_{y}^{p}\right)$. It follows from the assumptions and $(2.1)$ that:

$$
O p\left(a_{\lambda}\right)=\left(\begin{array}{cc}
P(h)-\lambda & u_{1} \\
\left\langle., u_{1}\right\rangle_{y} & 0
\end{array}\right)
$$

is $h$-pseudodifferential operator from $e^{-\psi(x) / h} S^{m}\left(\Omega, H^{2}\left(\mathbb{R}_{y}^{p}\right)\right)$ into $e^{-\psi(x) / h} S^{m}\left(\Omega, L^{2}\left(\mathbb{R}_{y}^{p}\right)\right)$, with operator valued symbol $a_{\lambda}$, where $\psi(x)$ is the Agmon distance associated to the metric $\lambda_{1}(x) d x^{2}$.

We now describe a method for finding the inverse of $O p\left(a_{\lambda}\right)$. Using the fact that $(\nabla \psi)^{2}(x)=\lambda_{1}(x)$ and the gap assumption (2.4), one can easily show that for $|\lambda|$ small enough and $\xi$ close enough to $i \nabla \psi(x)$,

$$
R e(\widehat{\Pi}(x) Q(x) \widehat{\Pi}(x)-\lambda)>0
$$

and $a_{\lambda}$ is invertible with inverse:

$$
b_{0}(x, \xi ; \lambda)=\left(\begin{array}{cc}
\widehat{\Pi}(x)\left(\xi^{2}+\widehat{\Pi}(x) Q(x) \widehat{\Pi}(x)-\lambda\right)^{-1} \widehat{\Pi}(x) & u_{1} \\
\left\langle., u_{1}\right\rangle_{y} & \lambda-\xi^{2}-\lambda_{1}(x)
\end{array}\right)
$$

where $\widehat{\Pi}(x)=1-\Pi(x)$ (see e.g. [3]).

In particular,

$$
b_{0}(x, \xi ; \lambda) \in S^{0}\left(\Omega^{*}, \mathcal{L}\left(L^{2}\left(\mathbb{R}_{y}^{p}\right) \oplus \mathbb{C}, H^{2}\left(\mathbb{R}_{y}^{p}\right) \oplus \mathbb{C}\right)\right)
$$

Then using the composition formula (2.2), it is easy to construct a symbol:

$$
\begin{aligned}
& b_{\lambda}(x, \xi ; h)=b_{0}(x, \xi ; \lambda)+h b_{1}(x, \xi ; \lambda)+h^{2} b_{2}(x, \xi ; \lambda)+\ldots \\
& b_{\lambda}(x, \xi ; h) \in S^{0}\left(\Omega^{*}, \mathcal{L}\left(L^{2}\left(\mathbb{R}_{y}^{p}\right) \oplus \mathbb{C}, H^{2}\left(\mathbb{R}_{y}^{p}\right) \oplus \mathbb{C}\right)\right)
\end{aligned}
$$


such that

$$
\begin{aligned}
a_{\lambda} \sharp b_{\lambda}(x, \xi ; h) & =1 \\
O p\left(a_{\lambda}\right) \circ O p\left(b_{\lambda}\right) & =I
\end{aligned}
$$

$I$ is the identity operator on $e^{-\psi(x) / h} S^{m}\left(\Omega, L^{2}\left(\mathbb{R}_{y}^{p}\right) \oplus \mathbb{C}\right)$. Let us pose:

$$
O p\left(b_{\lambda}\right)=\left(\begin{array}{cc}
E(\lambda) & E_{+}(\lambda) \\
E_{-}(\lambda) & E_{\mp}(\lambda)
\end{array}\right) .
$$

By Lemma 3.1 in [3], we also know that $E_{\mp}(\lambda)=O p\left(e_{\lambda}(x, \xi ; \lambda)\right)$ is $h$-pseudodifferential operator with symbol $e_{\lambda}(x, \xi ; \lambda) \in S^{0}\left(\Omega^{*}, \mathbb{C}\right)$ and its principal symbol is $e_{0}(x, \xi ; \lambda)=\lambda-\xi^{2}-\lambda_{1}(x)$. In particular, $F(\lambda)=$ $\lambda-E_{\mp}(\lambda)$ is a scalar $h$-pseudodifferential operator with principal symbol $\xi^{2}+\lambda_{1}(x)$. Moreover, we have the following fundamental spectral reduction:

$$
\lambda \in \sigma(P(h)) \Longleftrightarrow \lambda \in \sigma(F(\lambda))
$$

Hence, the spectral study of the Hamiltonian $P(h)$ on $L^{2}\left(\mathbb{R}_{x}^{n} \times \mathbb{R}_{y}^{p}\right)$ is reduced to that of the $h$ pseudodifferential operator $F(\lambda)$ on $L^{2}\left(\mathbb{R}_{x}^{n}\right)$ so-called effective Hamiltonian of $P(h)$. Now use Theorem 2.1 with $F(\lambda),|\lambda|$ small enough, we find BKW solutions of $P(h)$ as formal series $E_{k}(h)=e_{k} h+\sum_{j=1}^{\infty} e_{k, j} h^{1+j / 2}$ and $a_{k} \in S^{m_{k}}(\Omega, \mathbb{C})$, such that:

$$
\left(F\left(E_{k}(h)\right)-E_{k}(h)\right)\left(e^{-\psi(x) / h} a_{k}\right)=0
$$

in the exponentially weighted symbol space $e^{-\psi(x) / h} S^{m_{k}}(\Omega, \mathbb{C})$.

In fact, one can show in many situations that $F(\lambda)=P_{\text {eff }}(h)+\mathcal{O}\left(h^{2}\right)$, which makes it easy to compare (using, for example, the maximum principle) the eigenvalues of $P(h)$ and those of $P_{\text {eff }}(h)$, and then identify them when $h$ decays to zero fast enough [6]. This reduction will justify in the next section our definition of the normal Birkhoff forms of $P(h)$ as those of the effective Hamiltonian $P_{e f f}(h)$.

\section{Reduction to Birkhoff nORMal Form For the EFFECtive Hamiltonian}

There exists a very convenient way of constructing a canonical transformation such that we conserve the Hamiltonian structure of $P(h)$ by using the Birkhoff normal form theorem via the effective Hamiltonian $P_{\text {eff }}(h)$.

Definition 3.1. We call normal forms of the semi-classical operator $P(h)$, the Birkhoff normal forms of the associated effective Hamiltonian $P_{\text {eff }}(h)$.

The general philosophy will consist in transforming $P_{\text {eff }}(h)$ in such a way that the new Hamiltonian becomes $\hat{H}_{2}+\Lambda$ where $\hat{H}_{2}$ is the harmonic oscillator and $\Lambda$ is a reasonable perturbation term who commut 
with $\hat{H}_{2}$. We consider here $\Omega=\mathbb{R}_{x}^{n}$ and assume that the hessian matrix $\lambda_{1}^{\prime \prime}(0)$ is diagonal, let $\left(2 \nu_{1}^{2}, \ldots, 2 \nu_{n}^{2}\right)$ be its eigenvalues, with $\nu_{j}>0$ and $\nu=\left(\nu_{1}, \ldots, \nu_{n}\right)$. The rescaling $x_{j} \rightarrow \sqrt{\nu_{j}} x_{j}, x=\left(x_{1}, \ldots, x_{n}\right)$, transforms $P(h)$ as well as $P_{\text {eff }}(h)$ into:

$$
P_{e f f}(h)=\hat{H}_{2}+\Gamma(x)
$$

where $\hat{H}_{2}$ is the harmonic oscillator $\sum_{j=1}^{n} \nu_{j}\left(-h^{2} \frac{\partial^{2}}{\partial x_{j}^{2}}+x_{j}^{2}\right)$ and $\Gamma(x)$ is a smooth function such that $\Gamma(x)=\mathcal{O}\left(|x|^{3}\right)$ as $|x| \rightarrow 0$. In general, $\Gamma$ does not commute with $\hat{H}_{2}$, on the other hand we do not have enough information on this perturbation, for that we will use the Birkhoff normal form of $P(h)$ which is a transformation of the previous type but more adapted and less restrictive.

Let:

$$
S^{d}(m)=\left\{\begin{array}{l}
\left.\left.a(x, \xi ; h): \mathbb{R}_{x}^{n} \times \mathbb{R}_{\xi}^{n} \times\right] 0,1\right] \longrightarrow \mathbb{C}, \text { depends smoothly on } x \text { and } \xi \text { and } \\
\text { for all } \alpha \in \mathbb{N}^{2 n},\left|\partial_{(x, \xi)}^{\alpha} a(x, \xi ; h)\right| \leq C_{\alpha} h^{d}\left(1+|x|^{2}+|\xi|^{2}\right)^{m / 2}, \\
C_{\alpha}>0, \text { uniformly with respect } x, \xi \text { and } h
\end{array}\right\}
$$

where $m, d \in \mathbb{R} . S^{d}(m)$ is called the semiclassical space of symbols of order $d$ and degree $m$.

For $a \in S^{d}(m)$ and $u \in C_{0}^{\infty}\left(\mathbb{R}^{2 n}\right)$, we set:

$$
\begin{aligned}
(A u)(x) & =\left(O p_{\hbar}(a) u\right)(x) \\
& =(2 \pi h)^{-n} \int_{\mathbb{R}^{2 n}} e^{i h^{-1}\left\langle x-x^{\prime}, \xi\right\rangle} a\left(\frac{x+x^{\prime}}{2}, \xi ; h\right) u\left(x^{\prime}\right) d x^{\prime} d \xi
\end{aligned}
$$

$A$ is unbounded linear operator on $L^{2}\left(\mathbb{R}^{n}\right)$ with domain $C_{0}^{\infty}\left(\mathbb{R}^{2 n}\right)$ the space of infinitely differentiable functions on $\mathbb{R}^{2 n}$ with compact support, $A: C_{0}^{\infty}\left(\mathbb{R}^{2 n}\right) \longrightarrow C^{\infty}\left(\mathbb{R}^{2 n}\right)$ is called a semiclassical pseudodifferential operator with $h$-Weyl symbol $a$ of order $d$ and degree $m . \Psi^{d}\left(m, \mathbb{R}^{n}\right)$ denotes the set of all semiclassical pseudodifferential operators with symbols in the class $S^{d}(m)$.

Different classes of symbols can also be defined, but for our purpose this class is enough. For example, the $h$-Weyl symbol of the harmonic oscillator $\hat{H}_{2}$ is the polynomial $H_{2}=\sum_{j=1}^{n} \nu_{j}\left(\xi_{j}^{2}+x_{j}^{2}\right)$.

Now, we introduce the space $\mathcal{S}$ to be the set of formal series:

$$
\mathcal{S}=\left\{\sum_{\alpha, \beta \in \mathbb{N}^{n}, \ell \in \mathbb{N}} t_{\alpha, \beta, l} x^{\alpha} \xi^{\beta} h^{\ell}: t_{\alpha, \beta, l} \in \mathbb{C} \text { for all } \alpha, \beta \in \mathbb{N}^{n}, \ell \in \mathbb{N}\right\}
$$

where the degree of $x^{\alpha} \xi^{\beta} h^{\ell}$ is defined by $|\alpha|+|\beta|+2 \ell, \alpha, \beta \in \mathbb{N}^{n}, \ell \in \mathbb{N}$, for technical reasons that of $h$ is double-counted. Let $N \in \mathbb{N}$. Let $\mathcal{D}_{N}$ be the finite dimensional vector space:

$$
\mathcal{D}_{N}=\left\{\sum_{\alpha, \beta \in \mathbb{N}^{n}, \ell \in \mathbb{N}} t_{\alpha, \beta, l} x^{\alpha} \xi^{\beta} h^{\ell}: t_{\alpha, \beta, l} \in \mathbb{C}, \alpha, \beta \in \mathbb{N}^{n}, \ell \in \mathbb{N} \text { such that }|\alpha|+|\beta|+2 \ell=N\right\}
$$

and

$$
\mathcal{O}_{N}=\left\{\sum_{\alpha, \beta \in \mathbb{N}^{n}, \ell \in \mathbb{N}} t_{\alpha, \beta, l} x^{\alpha} \xi^{\beta} h^{\ell}: t_{\alpha, \beta, l}=0 \text { if }|\alpha|+|\beta|+2 \ell<N\right\}
$$


Note that, for all $N \in \mathbb{N}, \mathcal{D}_{N}$ and $\mathcal{O}_{N}$ are subspaces of $\mathcal{S}$ and

$$
\mathcal{S}=\mathcal{O}_{0} \supset \mathcal{O}_{1} \supset, \ldots, \bigcap_{N} \mathcal{O}_{N}=\{0\}
$$

Let $\langle., .\rangle_{W}$ be the Weyl bracket defined on $\mathcal{S}$ by:

$$
\langle f, g\rangle_{W}=\widehat{f} \widehat{g}-\widehat{g} \widehat{f}
$$

where $\widehat{f}$ and $\widehat{g}$ are the $h$-Weyl quantizations of symbols $f$ and $g$, respectively. Precisely,

$$
\left\langle f_{T}, g_{T}\right\rangle_{W}=\sigma_{W}\left(\langle f, g\rangle_{W}\right)=\sigma_{W}(\widehat{f} \widehat{g}-\widehat{g} \widehat{f})
$$

where $f_{T}$ and $g_{T}$ are formal Taylor series at the origin of $f$ and $g$ in $\mathcal{S}$, respectively and $\sigma_{W}$ denotes the $h$-Weyl symbol. Then, $\langle., .\rangle_{W}$ is antisymmetric satisfying the Jacobi identity:

$$
\left\langle\left\langle f_{T}, g\right\rangle_{W}, h_{T}\right\rangle_{W}+\left\langle\left\langle h_{T}, f_{T}\right\rangle_{W}, g_{T}\right\rangle_{W}+\left\langle\left\langle g_{T}, h_{T}\right\rangle_{W}, f_{T}\right\rangle_{W}=0
$$

and the Leibniz identity:

$$
\left\langle f_{T}, g_{T} h_{T}\right\rangle_{W}=\left\langle f_{T}, g_{T}\right\rangle_{W} h_{T}+g_{T}\left\langle f_{T}, h_{T}\right\rangle_{W}
$$

Thus, $\mathcal{S}$ equipped with the Weyl bracket is a Lie algebra such that:

$$
\begin{aligned}
\left\langle h, x_{j}\right\rangle_{W} & =\left\langle h, \xi_{j}\right\rangle_{W}=0 \text { and }\left\langle\xi_{j}, x_{j}\right\rangle_{W}=-i h, \text { for all } j=1, \ldots, n \\
x & =\left(x_{1}, \ldots, x_{n}\right) \text { and } \xi=\left(\xi_{1}, \ldots, \xi_{n}\right) \in \mathbb{R}^{n} .
\end{aligned}
$$

For and any $S \in \mathcal{S}$, we define a map:

$$
\begin{aligned}
a d_{S} \quad: \quad & \mathcal{S} \longrightarrow \mathcal{S} \\
& P \mapsto a d_{S}(P)=\langle S, P\rangle_{W}
\end{aligned}
$$

which is called the adjoint action. $\mathcal{S}$ has a representation on itself, the adjoint representation defined via the map ad.

Let us consider the important special case of this concept, which is the adjoint action $a d_{S}$ for $S \in \mathcal{D}_{2}$, and especially $a d_{H_{2}}$. Let $\mathbb{C}[z, \bar{z}, h]$ be the $\mathbb{C}$-linear space of polynomials spanned by $z^{\alpha} \bar{z}^{\beta} h^{\ell}$ of degree $|\alpha|+|\beta|+2 \ell$; $\alpha, \beta \in \mathbb{N}^{n}, \ell \in \mathbb{N}$, where $z=\left(x_{1}+i \xi_{1}, \ldots, x_{n}+i \xi_{n}\right) \in \mathbb{C}^{n}$ and $\bar{z}$ is the complex conjugate of $z$. Then, $\mathcal{B}=\left\{z^{\beta} \bar{z}^{\gamma}: z \in \mathbb{C}^{n} ; \beta, \gamma \in \mathbb{N}^{n}\right\}$ is a natural basis of $\mathbb{C}[z, \bar{z}, h]$.

The next proposition gives some important properties and results on $a d_{H_{2}}$.

Proposition 3.1. ([7]) 1) $i h^{-1} a d_{H_{2}}(P)=\left\{H_{2}, P\right\}$, where $\left\{H_{2}, P\right\}=\sum_{j=1}^{n} \frac{\partial H_{2}}{\partial \xi_{j}} \frac{\partial P}{\partial x_{j}}-\frac{\partial H_{2}}{\partial x_{j}} \frac{\partial P}{\partial \xi_{j}}$ is the classical Poisson bracket.

2) $a d_{H_{2}}$ is diagonal on $\mathcal{B}$, in the sense that $a d_{H_{2}}\left(z^{\beta} \bar{z}^{\gamma}\right)=h\langle\gamma-\beta, \nu\rangle z^{\beta} \bar{z}^{\gamma}$. 
The assumption (2.3) implies that $\lambda_{1}(x) \in \mathcal{O}_{3}$, and since $H_{2}+\lambda_{1}(x) \in \mathcal{D}_{2}$, the quantum Birkhoff normal form theorem for $P_{\text {eff }}(h)$ can now be formulated as follows:

Theorem 3.1. For $R \in \mathcal{O}_{3}$, there exist $S$ and $T$ in the subspace $\mathcal{O}_{3}$ with real coefficients such that:

$$
e^{i h^{-1} a d_{S}}\left(H_{2}+R\right)=H_{2}+T
$$

where $T=T_{3}+T_{4}+\ldots$ and $T_{j} \in \mathcal{D}_{j}$ commutes with $H_{2}:\left\langle H_{2}, T\right\rangle_{W}=0$.

This result is a direct consequence of the Birkoff normal form theorem shown for example in the article by Ghomari and Messirdi [7].

We gain, compared with the BKW constructions developed in the second section, the commutative property for Weyl product between the harmonic oscillator and the rest of reduction in Birkhoff normal form of the Hamiltonian. The Birkhoff normal form is a more usable semi-classical reduction involving other interesting spectral properties, especially it conserve the Hamiltonian structure and contains enough informations to study the quantum resonances.

\section{Birkhoff nORMAL FORM FOR $P(h)$ In FERMi RESONANCE}

It has been established in the previous sections that $P(h)$ can be reduced to the effective Hamiltonian $P_{\text {eff }}(h)$ modulo $\mathcal{O}\left(h^{2}\right)$. Thus, it is natural to define the Birkhoff normal forms of $P(h)$ as those of $P_{e f f}(h)$ modulo $\mathcal{O}\left(h^{2}\right)$.

Let us recall the definitions of the different relations of resonances for the frequencies $\left(\nu_{1}, \ldots, \nu_{n}\right)$ of $\hat{H}_{2}$ associated with the eigenvalues of the matrix $\lambda_{1}^{\prime \prime}(0)$. We say that the frequencies $\left(\nu_{1}, \ldots, \nu_{n}\right)$ are resonant if they are dependent over $\mathbb{Z}$, i.e., there exist integers $d_{1}, \ldots, d_{n} \in \mathbb{Z}$, not all zero, such that $d_{1} \nu_{1}+\ldots+d_{n} \nu_{n}=0$.

The number $d=\sum_{j=1}^{n}\left|d_{j}\right|$ is called the degree of resonance of $P_{\text {eff }}(h)$. In the particular resonant case where $\nu_{j}=\nu_{c} d_{j}$ for all $j=1, \ldots, n$ with $\nu_{c}>0$ and $d_{1}, \ldots, d_{n} \in \mathbb{N},\left(\nu_{j}\right)_{j}$ are said to be completely resonant.

As an application we study the structure of Birkhoff normal form in Fermi resonance $\left(\nu_{j}, 2 \nu_{j}\right)$. We compute the Birkhoff normal form of $P_{\text {eff }}(h)$ in the case of $1: 2$ resonance. Fermi resonances provide an essential mechanism for intramolecular vibrational energy flow and often dominate the vibrational dynamics in highly excited molecules. First discovered for the CO2 molecule, Fermi resonances are seen for many molecules.

Fermi resonance. The harmonic oscillator in Fermi resonance is given by:

$$
\widehat{H}_{2}=\left(-h^{2} \frac{\partial^{2}}{\partial x_{1}^{2}}+x_{1}^{2}\right)+2\left(-h^{2} \frac{\partial^{2}}{\partial x_{2}^{2}}+x_{2}^{2}\right)
$$


with symbol $H_{2}=\left|z_{1}\right|^{2}+2\left|z_{2}\right|^{2}$ where $z_{j}=x_{j}+i \xi_{j}, j=1,2$. We construct $K_{3} \in \mathcal{D}_{3}$ such that $\left\langle H_{2}, K_{3}\right\rangle_{W}=0$, so

$$
K_{3}=\sum_{2 \ell+|\alpha|+|\beta|=3} h^{\ell} z^{\alpha} \bar{z}^{\beta}
$$

with $\langle\nu, \beta-\alpha\rangle=0$. Thus, $K_{3}$ is generated by the monomials $z_{1}^{2} \bar{z}_{2}$ and $z_{2} \bar{z}_{1}^{2}$ and since $K_{3}$ is real, we can write:

$$
K_{3}=\rho \operatorname{Re}\left(z_{1}^{2} \bar{z}_{2}\right), \rho \in \mathbb{R} .
$$

Consequently, the Birkhoff normal form of the effective hamiltonian $P_{\text {eff }}(h)$ in the Fermi resonance, $\mathrm{H}_{2}+\mathrm{W}$ is equal to $\mathrm{H}_{2}+K_{3}+\mathcal{O}_{4}$, with

$$
\rho=\frac{1}{2 \sqrt{2}} \frac{\partial^{3} W}{\partial x_{1}^{2} \partial x_{2}}(0)
$$

see [8]. The Weyl quantization $\widehat{K}_{3}$ of $K_{3}$ is given by:

$$
\begin{aligned}
\widehat{K}_{3} & =\rho O p_{h}\left(\operatorname{Re}\left(z_{1}^{2} \bar{z}_{2}\right)\right)=\rho O p_{h}\left(x_{1}^{2} x_{2}+2 x_{1} \xi_{1} \xi_{2}-\xi_{1}^{2} x_{2}\right) \\
& =\rho\left[x_{1}^{2} x_{2}-h^{2}\left(2 x_{1} \frac{\partial^{2}}{\partial x_{1} \partial x_{2}}-x_{2} \frac{\partial^{2}}{\partial x_{1}^{2}}+\frac{\partial}{\partial x_{2}}\right)\right]
\end{aligned}
$$

and finally,

$$
\begin{aligned}
\widehat{H}_{2}+\widehat{K}_{3}= & \left(-h^{2} \frac{\partial^{2}}{\partial x_{1}^{2}}+x_{1}^{2}\right)+2\left(-h^{2} \frac{\partial^{2}}{\partial x_{2}^{2}}+x_{2}^{2}\right) \\
& +\rho\left[x_{1}^{2} x_{2}-h^{2}\left(2 x_{1} \frac{\partial^{2}}{\partial x_{1} \partial x_{2}}-x_{2} \frac{\partial^{2}}{\partial x_{1}^{2}}+\frac{\partial}{\partial x_{2}}\right)\right] .
\end{aligned}
$$

Acknowledgements: This work is supported by Laboratory of Fundamental and Applicable Mathematics of Oran (LMFAO) and is dedicated to Professor Bekkai Messirdi on the occasion of his 61th birthday.

Conflicts of Interest: The author(s) declare that there are no conflicts of interest regarding the publication of this paper.

\section{REFERENCES}

[1] H. Baklouti, Asymptotic expansion for the widths of resonances in Born-Oppenheimer approximation, Asymptot. Anal. 69(1-2) (2010), 1-29.

[2] A. Balazard-Konlein, Asymptotique semi-classique du spectre pour des opérateurs à symbole operatoriel, C. R. Acad. Sci. Paris Sér. I Math. 301 (1985), 903-906.

[3] S. Belmohoub and B. Messirdi, Singular Schrödinger Operators via Grushin Problem Method, Ann. Oradea Univ. Math. Fascicola. 24(1) (2017), 83-91.

[4] G.D. Birkhoff, Dynamical Systems, AMS Colloq. Publ. 9, AMS New York. (1927).

[5] M. Born and R. Oppenheimer, Zur Quantentheorie der Molekeln, Ann. Physics. 84 (1927), 457-484.

[6] J.M. Combes and R. Seiler, Regularity and asymptotic properties of the discrete spectrum of electronic hamiltonians, Int.

J. Quantum Chem. 14 (1978), 213-229. 
[7] K. Ghomari and B. Messirdi, Quantum Birkhoff-Gustavson Normal Form in Some Completely Resonant Cases, J. Math. Anal. Appl. 378 (2011), 306-313.

[8] K. Ghomari and B. Messirdi, Hamiltonians Spectrum in Fermi Resonance via The Birkhoff-Gustavson Normal Form, Int. J. Contemp. Math. Sciences. 4(35) (2009), 1701-1707.

[9] B. Helffer and J. Sjöstrand, Multiple wells in the semiclassical limit I, Commun. Part. Diff. Equ. 9(4) (1984), 337-408.

[10] B. Messirdi, Asymptotique de Born-Oppenheimer pour la prédissociation moléculaire (cas de potentiels réguliers), Ann. Henri Poincaré (A). 61 (1992), 255-292.

[11] B. Messirdi and K. Ghomari, Resonances of a two-state semiclassical Schrödinger Hamiltonians, Appl. Anal. 86(2) (2007), $187-204$. 